\title{
Combination of Non-Invasive Fibrosis Tests: a Solution for the Prediction of Advanced Fibrosis in Non-Alcoholic Fatty Liver Disease?
}

\author{
Monica Lupşor-Platon
}

Department of Medical

Imaging, Iuliu Haţieganu

University of Medicine and

Pharmacy,

Prof. Dr. Octavian Fodor

Regional Institute of

Gastroenterology and

Hepatology, Cluj-Napoca,

Romania

\author{
Address for correspondence: \\ Monica Lupsor-Platon \\ Department of Medical \\ Imaging, Iuliu Hatieganu \\ University of Medicine and \\ Pharmacy, \\ Regional Institute of \\ Gastroenterology and \\ Hepatology, Cluj-Napoca, \\ 19-21 Croitorilor Street, \\ 400162 Cluj-Napoca, Romania \\ monica.lupsor@umfcluj.ro
}

Received: 11.08.2019

Accepted: 15.08.2019
An increasingly common cause of chronic liver disease in adults and children is nonalcoholic fatty liver disease (NAFLD) [1]. According to the EASL-EASD-EASO Clinical Practice Guidelines [2], NAFLD is characterized by excessive hepatic fat accumulation, associated with insulin resistance, and is defined by the presence of steatosis in $>5 \%$ of the hepatocytes. This proportion is quantified by histological analysis, or by a proton density fat fraction $>5.6 \%$, assessed with proton magnetic resonance spectroscopy (1HMRS) or quantitative fat/water selective magnetic resonance imaging (MRI) [2].

Non-alcoholic fatty liver disease may present in various ways: as simple steatosis, nonalcoholic steatohepatitis, liver cirrhosis, or even hepatocellular carcinoma (HCC) [3-6]. Of all the pathologic changes found in NAFLD, it appears that fibrosis alone is independently associated with long-term overall mortality, liver transplantation, and liver-related events (i.e. cirrhosis, liver failure and portal hypertension) [7], which makes the quantification of this parameter even more important.

For the moment, the gold standard in the assessment of fibrosis is biopsy. This is an invasive method, with potential adverse effects and great interand intra-observer variability [8-10]. In addition, we must not forget that in NASH patients, the histopathologic assessment may be flawed due to the inhomogeneous distribution of fibrosis. Ratziu et al. [10] analyzed two samples of the right liver lobe in all patients and found agreement regarding fibrosis stage in only $47 \%$ of patients, while differences of at least 1 or 2 stages were found in $41 \%$ and $12 \%$ of cases, respectively [10]. As a result, research is increasingly focused on finding alternative, non-invasive techniques for the assessment of fibrosis. As mentioned in EASL-ALEH Clinical Practice Guidelines, the non-invasive tests for evaluation of liver disease severity and prognosis are based on two approaches: a "physical" one (the measurement of liver stiffness) and a "biological" one, relying on the quantification of serum markers. Although these approaches are complementary, they are based on different rationales [11].

As far as the physical approach is concerned, liver stiffness, measured using elastographic techniques, is a physical property, intrinsic to the hepatic parenchyma. Various studies, using mainly transient elastography (TE), have showed that liver stiffness is strongly correlated with fibrosis stage, but when compared with other categories of patients (such as those with chronic viral hepatitis) - the correlation is somewhat weaker in NASH patients [12]. This can be partly explained by the different distribution pattern in the two conditions. The accumulation of dense stellate portal fibrosis (present in viral hepatitis) will increase liver stiffness in direct proportion, while the perisinusoidal fibrosis distributed especially in the centrolobular areas (typical for NASH) will not induce a similar increase rate, as proven by morphometric studies [13]. According to the EFSUMB and EASL Guidelines and Recommendations on the Clinical Use of Liver Ultrasound Elastography $[11,14]$, TE can be used to confidently exclude severe fibrosis and especially cirrhosis in NAFLD patients, with a high negative predictive value (around 90\%). The main shortcoming of TE is that it provides unreliable results in patients with a high BMI and/or thoracic fold thickness [2]. Some of these situations may be prevented using the XL probe, thus decreasing the failure rate, which however remains significant in patients with a distance between the skin and liver capsule above $3.4 \mathrm{~cm}$ and in extremely obese patients (BMI > $\left.40 \mathrm{~kg} / \mathrm{m}^{2}\right)[15,16]$.

In what concerns the biological approach, a large number of biochemical markers related to inflammation, apoptosis and oxidative stress have also been reported to diagnose liver fibrosis 
in NAFLD patients [17-19], but these are insufficiently accurate when used alone [20,21]. Therefore, the models elaborated for the non-invasive diagnosis of fibrosis, including related clinical and biochemical indicators have become the focus of research in NAFLD, as well. Several clinical scoring systems have indeed been applied in diagnosing fibrosis, such as the aspartate aminotransferase (AST)-to-platelet ratio index (APRI), FIB-4, Forns' Index, the BARD score, FibroMeter NAFLD, the Hui model, the non-invasive Koeln-Essen-index (NIKEI), the S Index and the NAFLD fibrosis score (NFS) [22-29].

In this issue of the J Gastrointestin Liver Dis, Yang et al. [30] have assessed in 453 consecutive patients with biopsyproven NAFLD, the diagnostic performance of nine clinical non-invasive fibrosis models for advanced fibrosis prediction: APRI, FiB-4, Forns' Index, BARD, FibroMeter NAFLD, the Hui model, NIKEI, the S Index and the NAFLD fibrosis score (NFS). All the nine fibrotic models were correlated with the fibrosis stage in patients with NAFLD. Despite the high negative predictive value and specificity for diagnosing fibrosis of all these models, the positive predictive value and sensitivity were low. These results suggest that these models are more useful in excluding significant and advanced fibrosis in NAFLD, than in predicting their presence.

Although the highest area under the ROC curve and negative predictive value were found for APRI, BARD, FibroMeter NAFLD and NIKEI, the use of only one noninvasive model was not accurate enough to diagnose severe fibrosis in NAFLD. Conversely, the authors have proven that, using a step layered combination of APRI, BARD, FibroMeter NAFLD and NIKEI leads to an increased diagnostic performance for the prediction of advanced fibrosis in NAFLD, with $89.13 \%$ specificity, $72.50 \%$ sensitivity, $74.36 \%$ negative predictive value and $88.17 \%$ positive predictive value.

Undoubtedly, studies are still necessary before prediction models and blood-based biomarkers become available for routine clinical care in patients with NAFLD [31]. The future may bring more complex models, including clinical, routine blood-based variables, markers that reflect the dynamic nature of the fibrogenesis, alongside with molecular markers, quantitative proteomic technology, and physical parameters, such as liver stiffness assessed by elastographic techniques.

Conflicts of interest: None to declare.

\section{REFERENCES}

1. Younossi ZM, Blissett D, Blissett R, et al. The economic and clinical burden of nonalcoholic fatty liver disease in the United States and Europe. Hepatology 2016;64:1577-1586. doi:10.1002/hep.28785

2. Blond E, Disse E, Cuerq C, et al. EASL-EASD-EASO clinical practice guidelines for the management of non-alcoholic fatty liver disease in severely obese people:do they lead to over-referral? Diabetologia 2017;60:1218-1222. doi:10.1007/s00125-017-4264-9

3. Bang KB, Cho YK. Comorbidities and metabolic derangement of NAFLD. J Lifestyle Med 2015;5:7-13. doi:10.15280/jlm.2015.5.1.7

4. Cho Y, Tokuhara D, Morikawa H, et al. Transient elastography- based liver profiles in a hospital-based pediatric population in Japan. PLoS One 2015;10:e0137239. doi:10.1371/journal.pone.0137239
5. Byrne CD, Targher G. NAFLD: a multisystem disease. J Hepatol 2015;62(1 Suppl):S47-S64. doi:10.101 6/j.jhep.2014.12.012

6. Smits MM, Ioannou GN, Boyko EJ, Utzschneider KM. Non-alcoholic fatty liver disease as an independent manifestation of the metabolic syndrome: results of a US national survey in three ethnic groups. J Gastroenterol Hepatol 2013;28:664-670. doi:10.1111/jgh.12106

7. Angulo P, Kleiner DE, Dam-Larsen S, et al. Liver Fibrosis, but No Other Histologic Features, Is Associated With Long-term Outcomes of Patients With Nonalcoholic Fatty Liver Disease. Gastroenterology 2015;149:389-397.e10. doi:10.1053/j.gastro.2015.04.043

8. Bedossa $\mathrm{P}$, Dargère D, Paradis V. Sampling variability of liver fibrosis in chronic hepatitis C. Hepatology. 2003;38:1449-1457. doi:10.1016/j. hep.2003.09.022

9. Regev A, Berho M, Jeffers LJ, et al. Sampling error and intraobserver variation in liver biopsy in patients with chronic HCV infection. Am J Gastroenterol 2002;97:2614-2618.

10. Ratziu V, Charlotte F, Heurtier A, et al. Sampling variability of liver biopsy in nonalcoholic fatty liver disease. Gastroenterology 2005;128:1898-1906. doi:10.1053/j.gastro.2005.03.084

11. European Association for Study of Liver; Asociacion Latinoamericana para el Estudio del Higado. EASL-ALEH Clinical Practice Guidelines: Non-invasive tests for evaluation of liver disease severity and prognosis. J Hepatol 2015;63:237-264. doi:10.1016/j.jhep.2015.04.006

12. Lupsor M, Badea R, Stefanescu H, et al. Performance of unidimensional transient elastography in staging non-alcoholic steatohepatitis. J Gastrointestin Liver Dis 2010;19:53-60.

13. Ziol M, Kettaneh A, Ganne-Carrié N, Barget N, Tengher-Barna I, Beaugrand M. Relationships between fibrosis amounts assessed by morphometry and liver stiffness measurements in chronic hepatitis or steatohepatitis. Eur J Gastroenterol Hepatol 2009;21:1261-1268. doi:10.1097/MEG.0b013e32832a20f5

14. Dietrich CF, Bamber J, Berzigotti A, et al. EFSUMB Guidelines and Recommendations on the Clinical Use of Liver Ultrasound Elastography, Update 2017 (Long Version). Ultraschall Med 2017;38:e16-e47. doi:10.1055/s-0043-103952

15. Myers RP, Pomier-Layrargues G, Kirsch R, et al. Feasibility and diagnostic performance of the FibroScan XL probe for liver stiffness measurement in overweight and obese patients. Hepatology 2012;55:199-208. doi:10.1002/hep.24624

16. Lupsor-Platon M. Noninvasive Evaluation of Fibrosis and Steatosis in Nonalcoholic Fatty Liver Disease by Elastographic Methods. In: Rodrigo Valenzuela (ed). Non-Alcoholic Fatty Liver Disease - Molecular Bases, Prevention and Treatment 2018, UK, In-Tech:93-117.

17. Yoneda M, Mawatari H, Fujita K, et al. High-sensitivity C-reactive protein is an independent clinical feature of nonalcoholic steatohepatitis (NASH) and also of the severity of fibrosis in NASH. J Gastroenterol 2007;42:573-582. doi:10.1007/s00535-007-2060-x

18. Wieckowska A, Papouchado BG, Li Z, Lopez R, Zein NN, Feldstein AE. Increased hepatic and circulating interleukin-6 levels in human nonalcoholic steatohepatitis. Am J Gastroenterol 2008;103:1372-1379.

19. Nazal L, Riquelme A, Solís N, et al. Hypoadiponectinemia and its association with liver fibrosis in morbidly obese patients. Obes Surg 2010;20:1400-1407. doi:10.1007/s11695-009-0051-0

20. Torres DM, Harrison SA. Diagnosis and therapy of nonalcoholic steatohepatitis. Gastroenterology 2008;134:1682-1698. doi:10.1053/j. gastro.2008.02.077

21. Lewis JR, Mohanty SR. Nonalcoholic fatty liver disease: a review and update. Dig Dis Sci 2010;55:560-578. doi:10.1007/s10620-0091081-0 
22. Wai CT, Greenson JK, Fontana RJ, et al. A simple noninvasive index can predict both significant fibrosis and cirrhosis in patients with chronic hepatitis C. Hepatology 2003;38:518-526. doi:10.1053/jhep.2003.50346

23. Harrison SA, Oliver D, Arnold HL, Gogia S, Neuschwander-Tetri BA. Development and validation of a simple NAFLD clinical scoring system for identifying patients without advanced disease. Gut 2008;57:14411447. doi:10.1136/gut.2007.146019

24. Sterling RK, Lissen E, Clumeck N, et al. APRICOT Clinica Investigators:Development of a simple noninvasive index to predict significant fibrosis in patients with $\mathrm{HIV} / \mathrm{HCV}$ coinfection. Hepatology 2006;43:1317-1325. doi:10.1002/hep.21178

25. Vallet-Pichard A, Mallet V, Nalpas B, et al. FIB-4: an inexpensive and accurate marker of fibrosis in HCV infection. comparison with liver biopsy and fibrotest. Hepatology 2007;46:32-36. doi:10.1002/hep.21669

26. Calès P, Lainé F, Boursier J, et al. Comparison of blood tests for liver fibrosis specific or not to NAFLD. J Hepatol 2009;50:165-173. doi:10.1016/j.jhep.2008.07.035
27. Forns X, Ampurdanès S, Llovet JM, et al. Identification of chronic hepatitis $\mathrm{C}$ patients without hepatic fibrosis by a simple predictive model. Hepatology 2002;36:986-992. doi:10.1053/jhep.2002.36128

28. Demir M, Lang S, Schlattjan M, et al. NIKEI: a new inexpensive and non-invasive scoring system to exclude advanced fibrosis in patients with NAFLD. PLoS One 2013;8:e58360. doi:10.1371/journal. pone. 0058360

29. Hui AY, Chan HL, Wong VW, et al. Identification of chronic hepatitis B patients without significant liver fibrosis by a simple noninvasive predictive model. Am J Gastroenterol 2005;100:616-623.

30. Yang M, Jiang L, Wang Y, et al. Step Layered Combination of Noninvasive Fibrosis Models Improves Diagnostic Accuracy of Advanced Fibrosis in Nonalcoholic Fatty Liver Disease. J Gastrointestin Liver Dis 2019;28(3):289-296 doi: 10.15403/jgld-420

31. Vilar-Gomez E, Chalasani N. Non-invasive assessment of non-alcoholic fatty liver disease:Clinical prediction rules and blood-based biomarkers. J Hepatol 2018;68:305-315. doi:10.1016/j.jhep.2017.11.013 